\title{
Pentagon Relations in Direct Sums and Grassmann Algebras
}

\author{
Igor G. KOREPANOV and Nurlan M. SADYKOV
}

Moscow State University of Instrument Engineering and Computer Sciences, 20 Stromynka Str., Moscow 107996, Russia

E-mail: paloff@ya.ru,nik56-74@mail.ru

Received December 19, 2012, in final form April 05, 2013; Published online April 10, 2013

http://dx.doi.org/10.3842/SIGMA.2013.030

\begin{abstract}
We construct vast families of orthogonal operators obeying pentagon relation in a direct sum of three $n$-dimensional vector spaces. As a consequence, we obtain pentagon relations in Grassmann algebras, making a far reaching generalization of exotic Reidemeister torsions.
\end{abstract}

Key words: Pachner moves; pentagon relations; Grassmann algebras

2010 Mathematics Subject Classification: 15A75; 57Q99; 57R56

\section{Introduction}

Pachner moves [12] are elementary local rebuildings of a manifold triangulation. There are $n+1$ types of Pachner moves in dimension $n$, and a triangulation of a piecewise-linear $n$-manifold can be transformed into another triangulation using a sequence of these moves; a pedagogical introduction in this theory can be found in [11]. In dimension three, the Pachner moves are:

- move $2 \rightarrow 3$, replacing two adjacent tetrahedra by three tetrahedra occupying the same place in the manifold,

- inverse move $3 \rightarrow 2$,

- move $1 \rightarrow 4$, decomposing a tetrahedron into four tetrahedra with a new vertex inside it,

- and the inverse move $4 \rightarrow 1$.

A move $2 \rightarrow 3$ is depicted in Fig. 1: the left-hand side consists of tetrahedra 1234 and 1345, while the right-hand side - of tetrahedra 1245, 2345 and 1235.

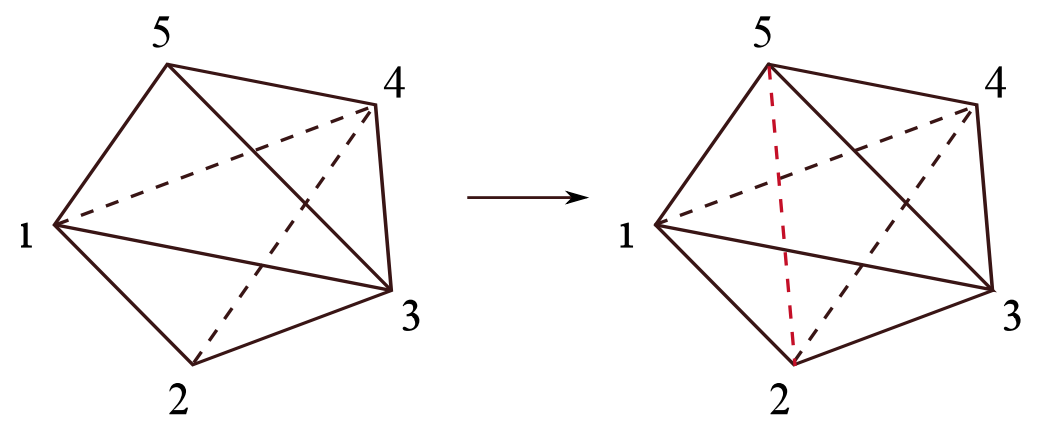

Figure 1. Pachner move $2 \rightarrow 3$.

We call pentagon relation any algebraic formula that corresponds naturally to a move $2 \rightarrow 3$, in such way that there is a hope to move further and develop a theory that would give some sort 
of manifold invariant. Specifically, this paper deals with pentagon relations in a direct sum of complex vector spaces, as in formulas (4) and (5) below (where matrix entries can be themselves matrices), and in a Grassmann algebra, as in formula (33). A subtle point is that a Grassmannalgebraic relation follows from a direct-sum relation if the latter is made of orthogonal operators, so the construction of such "orthogonal" relations, by means of ansatz (18), (21), involving some non-commutative algebra, is the central result of this paper.

Remark 1. Our pentagon relations are thus slightly unusual, because usually pentagon relations are written in the tensor product of vector spaces, see for instance [5] and [2]. Nevertheless, paper [5] already contains an example of a relation in direct sum, as we explain in the end of Section 3.

Remark 2. Experience shows that if an interesting formula corresponding to moves $2 \leftrightarrow 3$ has been found, then, usually, its counterpart corresponding to moves $1 \leftrightarrow 4$ can be found too.

Below,

- in Section 2, we explain how a pentagon relation in a direct sum follows if vector spaces are put in correspondence to plane polygons, with the condition that if two of them make together a larger polygon, then the two corresponding spaces are added directly,

- in the warming-up Section 3, we obtain, in our own way, the Kashaev's pentagon relation for orthogonal matrices in the direct sum of one-dimensional spaces,

- in Section 4, we present a simple general construction of vector spaces needed for pentagon relation, but without the requirement of orthogonality,

- in the central Section 5, we introduce an elegant Euclidian metric in these vector spaces,

- in Section 6, we look more closely at the case where the space attached to each triangle is two-dimensional, and the (complex) Euclidian metric is introduced in terms of isotropic vectors that will correspond to fermionic creation-annihilation operators,

- in Section 7, we briefly recall the fundamentals of Grassmann-Berezin calculus of anticommuting variables, and write out a Grassmann-algebraic pentagon relation,

- in Section 8, we write out formulas connecting a Grassmann-Gaussian exponent taken as a tetrahedron Grassmann weight, and an orthogonal matrix,

- and in Section 9, we show that our construction includes the earlier introduced Grassmann tetrahedron weights related to exotic Reidemeister torsions.

We plan to write one more paper, containing relations corresponding to moves $1-4$, construction of a TQFT, and calculations for specific manifolds.

\section{Pentagon relation in a direct sum of based vector spaces: generalities}

We now want to interpret our Fig. 1 as a flat figure - projection of the two sides of the Pachner move onto the plane. Thus, for instance, the back surface of two tetrahedra in the left-hand side of Fig. 1 becomes a triangulated pentagon 12345, made of three triangles 124, 234 and 145; this is also shown as the leftmost pentagon in Fig. 2 or 3 . We put in correspondence to this flat geometric picture the following algebraic objects. An $n$-dimensional complex vector space will correspond to each triangle with vertices in $\{1, \ldots, 5\}$, and we require the following condition.

Condition 1. If some triangles make together a triangulation of a greater polygon (quadrilateral or the whole pentagon), then the direct sum of the corresponding spaces depends only on this polygon, i.e., is the same for other triangulation(s). 

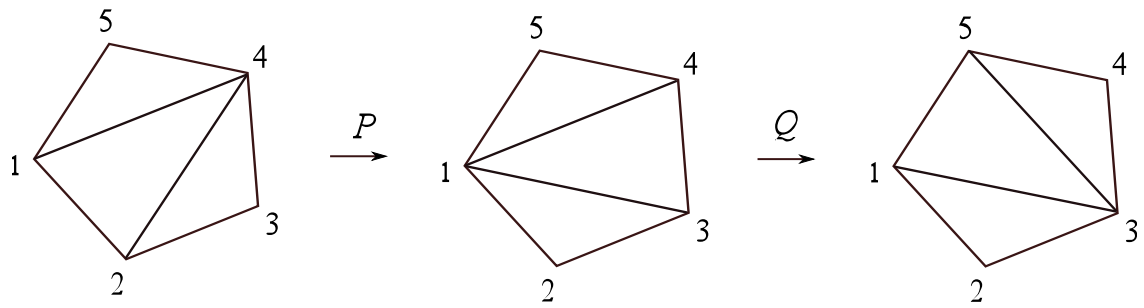

Figure 2. Flips in pentagon triangulation corresponding to the l.h.s. of Pachner move $2 \rightarrow 3$.

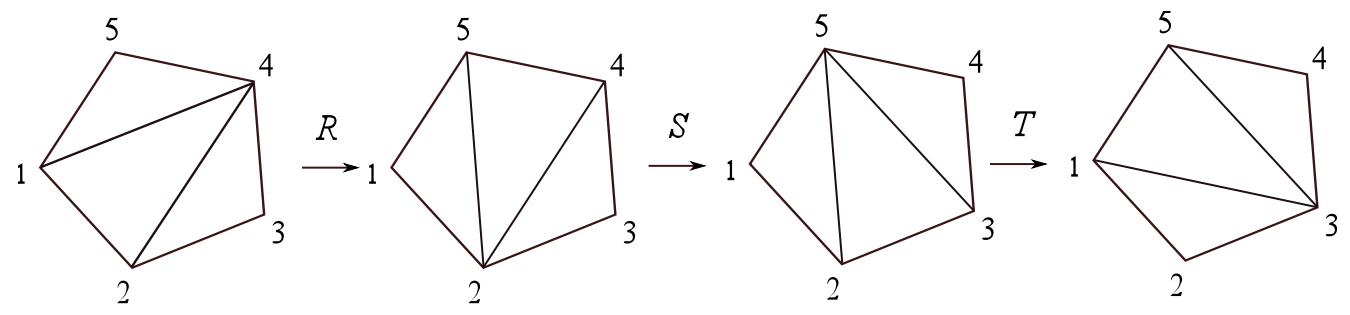

Figure 3. Flips in pentagon triangulation corresponding to the r.h.s. of Pachner move $2 \rightarrow 3$.

Thus, all convex quadrilaterals, as well as the pentagon 12345, acquire their vector spaces as well. Taking some liberty, we denote these spaces the same way as polygons, and write Condition 1 as

$$
1234=124 \oplus 234=123 \oplus 134, \quad 12345=124 \oplus 234 \oplus 145=\cdots, \quad \text { etc }
$$

We further assume that our spaces corresponding to triangles are based - equipped with chosen bases. The bases will be written as columns made of basis vectors, for instance,

$$
\mathbf{f}_{124}=\left(\begin{array}{c}
\mathbf{f}_{124}^{(1)} \\
\vdots \\
\mathbf{f}_{124}^{(n)}
\end{array}\right)
$$

For a greater polygon, any decomposition like those in (1) provides a basis in the form of the ordered union of bases in triangles, written as, for instance, $\left(\begin{array}{l}\mathbf{f}_{124} \\ \mathbf{f}_{234}\end{array}\right)$ if we are considering the decomposition $1234=124 \oplus 234$.

Remark 3. The specific order of bases in this union can be fixed in any convenient way. In the present paper, it always complies with the following rule: $\mathbf{f}_{i j k}$ goes before $\mathbf{f}_{i^{\prime} j^{\prime} k^{\prime}}$ if $i+j+k<$ $i^{\prime}+j^{\prime}+k^{\prime}$. Actually, this order arose when we were doing calculations in what we were seeing as the most natural way. We never meet here a situation where $i+j+k=i^{\prime}+j^{\prime}+k^{\prime}$.

Remark 4. The vertices of a triangle $i j k$ always go in this paper in the increasing order: $i<j<k$. Note that this order induces a consistent orientation of all triangles in all pentagons in Figs. 2 and 3. As we are dealing here only with five vertices and ten triangles, the orientation issues do not bring about any problems; we will, however, comment on their importance in a specific situation below in Remark 9.

The l.h.s. of Fig. 1 can be seen as the tetrahedra 1234 and 1345 glued to the back surface 12345 . In our flat picture, each tetrahedron corresponds to a flip, or two-dimensional Pachner move, in the triangulation of 12345; the sequence of these two flips is depicted in Fig. 2. 
We denote these flips $P$ and $Q$, and use the same notations for their corresponding matrices defined as the following change-of-basis matrices in 12345 :

$$
\left(\begin{array}{c}
\mathbf{f}_{124} \\
\mathbf{f}_{234} \\
\mathbf{f}_{145}
\end{array}\right) \stackrel{P}{\longmapsto}\left(\begin{array}{c}
\mathbf{f}_{123} \\
\mathbf{f}_{134} \\
\mathbf{f}_{145}
\end{array}\right) \stackrel{Q}{\longmapsto}\left(\begin{array}{c}
\mathbf{f}_{123} \\
\mathbf{f}_{135} \\
\mathbf{f}_{345}
\end{array}\right) .
$$

Sometimes it will be convenient for us to write this also in the following "matrix $\times$ basis" form:

$$
P\left(\begin{array}{l}
\mathbf{f}_{124} \\
\mathbf{f}_{234} \\
\mathbf{f}_{145}
\end{array}\right)=\left(\begin{array}{l}
\mathbf{f}_{123} \\
\mathbf{f}_{134} \\
\mathbf{f}_{145}
\end{array}\right), \quad Q\left(\begin{array}{l}
\mathbf{f}_{123} \\
\mathbf{f}_{134} \\
\mathbf{f}_{145}
\end{array}\right)=\left(\begin{array}{l}
\mathbf{f}_{123} \\
\mathbf{f}_{135} \\
\mathbf{f}_{345}
\end{array}\right) .
$$

Similarly, the r.h.s. of Fig. 1 can be seen as the tetrahedra 1245, 2345, and 1235 glued to the back surface 12345. In our flat picture, this corresponds to the sequence of three flips in the triangulation of 12345 depicted in Fig. 3. We denote these flips $R, S$ and $T$, and their corresponding matrices are determined by the following actions on bases of 12345 :

$$
\left(\begin{array}{l}
\mathbf{f}_{124} \\
\mathbf{f}_{234} \\
\mathbf{f}_{145}
\end{array}\right) \stackrel{R}{\longmapsto}\left(\begin{array}{l}
\mathbf{f}_{125} \\
\mathbf{f}_{234} \\
\mathbf{f}_{245}
\end{array}\right) \stackrel{S}{\longmapsto}\left(\begin{array}{l}
\mathbf{f}_{125} \\
\mathbf{f}_{235} \\
\mathbf{f}_{345}
\end{array}\right) \stackrel{T}{\longmapsto}\left(\begin{array}{l}
\mathbf{f}_{123} \\
\mathbf{f}_{135} \\
\mathbf{f}_{345}
\end{array}\right) .
$$

Theorem 1. Matrices $P, \ldots, T$ have the following forms:

$$
\begin{array}{rlrl}
P=\left(\begin{array}{lll}
* & * & 0 \\
* & * & 0 \\
0 & 0 & 1
\end{array}\right), & Q=\left(\begin{array}{lll}
1 & 0 & 0 \\
0 & * & * \\
0 & * & *
\end{array}\right), \\
R=\left(\begin{array}{lll}
* & 0 & * \\
0 & 1 & 0 \\
* & 0 & *
\end{array}\right), & S=\left(\begin{array}{lll}
1 & 0 & 0 \\
0 & * & * \\
0 & * & *
\end{array}\right), & T=\left(\begin{array}{lll}
* & * & 0 \\
* & * & 0 \\
0 & 0 & 1
\end{array}\right),
\end{array}
$$

where each zero, one or asterisk is an $n \times n$ matrix block, and the following pentagon relation in the direct sum holds:

$$
Q P=T S R \text {. }
$$

Proof. The form (4) of matrices is clear from (2) and (3), as well as the relation (5) that follows from the fact that both leftmost and rightmost bases in (2) and (3) are the same.

Remark 5. We can now forget about the space 12345 and its subspaces and consider matrices $P, \ldots, T$ simply as linear operators acting in the $3 n$-dimensional space of usual column vectors the direct sum of three such $n$-dimensional spaces. Each of the matrices $P, \ldots, T$ acts nontrivially in the direct sum of two such spaces, and can be regarded, in the spirit of similar relations in mathematical physics, as a $2 n \times 2 n$ matrix, identified when necessary with its direct sum with an identity matrix.

Remark 6. Equation (5) for matrices (4) can be regarded as a simplified version of "dynamical Yang-Baxter equation in a direct sum", introduced by one of the authors [7] in 1994. The latter equation appears at each step of evolution of an integrable dynamical system in discrete time, see [7, formulas (3), (4) and (10)], and proved its usefulness and fundamental character both in dynamical systems and constructing solutions to Zamolodchikov tetrahedron equation, enough to mention papers $[4,6,10,13,14]$.

Thus, if we construct $n$-dimensional subspaces of a $3 n$-dimensional vector space as described in the beginning of this section, including Condition 1 , we get matrices satisfying the pentagon relation in the direct sum. This turns out to be, in itself, quite easy; interesting complications come forward, as we will see, when there is additional requirement of orthogonality. 


\section{Kashaev's pentagon relation for orthogonal matrices in a direct sum of one-dimensional spaces}

In this section, we take the following space $V$ as the space 12345 introduced in Section 2: by definition, $V$ is the three-dimensional complex vector space of row vectors of length 5

$$
\mathbf{f}=\left(\begin{array}{lllll}
f_{1} & f_{2} & f_{3} & f_{4} & f_{5}
\end{array}\right)
$$

subject to the conditions

$$
f_{1}+f_{2}+f_{3}+f_{4}+f_{5}=0, \quad \zeta_{1} f_{1}+\zeta_{2} f_{2}+\zeta_{3} f_{3}+\zeta_{4} f_{4}+\zeta_{5} f_{5}=0,
$$

where $\zeta_{i}$ are some constants, all pairwise different. We will be using the following notation for their differences:

$$
\zeta_{i j} \stackrel{\text { def }}{=} \zeta_{i}-\zeta_{j} \text {. }
$$

Remark 7. Our motivation for introducing the vectors (6) with conditions (7) here, and their generalizations below, was simply that we have already used such objects in our earlier works, although in a different context. It is enough to mention the paper [8] and formulas (8), (9) and the unnumbered one, next after (33), therein.

For $1 \leq i<j<k \leq 5$, choose vectors $\mathbf{f}_{i j k}$ with only $i$-th, $j$-th and $k$-th nonzero components. This means that, for instance,

$$
\mathbf{f}_{135}=\operatorname{const}_{135}\left(\zeta_{35} \quad 0 \quad \zeta_{51} \quad 0 \quad \zeta_{13}\right) .
$$

Such a vector forms itself a basis in the one-dimensional space $i j k$, corresponding to the triangle $i j k$. At this stage, the nonzero constants const ${ }_{i j k}$ can be arbitrary.

Now we define $3 \times 3$ matrices $P, Q, R, S$ and $T$ by the conditions (2) and (3), and get a solution to (5) nicely parametrized by numbers $\zeta_{i}$. In particular, the matrix corresponding to a tetrahedron depends only on the four $\zeta_{i}$ 's at its vertices.

Next, we want to make orthogonal operators from our matrices $P, \ldots, T$. This is done in two steps. First, we introduce a scalar product between vectors (6), (7), and second, we choose constants const ${ }_{i j k}$, in such way that the following conditions hold.

Condition 2. Scalar product between vectors (6), (7) must be such that all three vectors in any column in formulas (2) and (3) become pairwise orthogonal.

Condition 3. The normalizing constants const ${ }_{i j k}$ are such that all $\mathbf{f}_{i j k}$ become unit vectors.

Condition 2 applies to vectors corresponding to triangles with disjoint interiors, i.e., any two triangles within any chosen pentagon in Figs. 2 and 3; it can be checked that Condition 2 for all such triangles follows from the five independent conditions

$$
123 \perp 345, \quad 234 \perp 145, \quad 345 \perp 125, \quad 145 \perp 123, \quad 125 \perp 234 .
$$

As a symmetric $3 \times 3$ matrix determining the (complex) Euclidian metric in our three-dimensional space $V$ has six independent entries, it is determined by the five linear conditions (9) uniquely up to a scalar factor. Then, Condition 3 determines uniquely all normalizers const ${ }_{i j k}$.

We do not present here explicit formulas for the scalar product and normalizing constants, as they are written out below (formulas (18) and (21)) in a more general case. The resulting pentagon relation for orthogonal matrices is:

$$
\left(\begin{array}{ccc}
1 & 0 & 0 \\
0 & \cos \varphi_{1345} & \sin \varphi_{1345} \\
0 & -\sin \varphi_{1345} & \cos \varphi_{1345}
\end{array}\right)\left(\begin{array}{ccc}
\cos \varphi_{1234} & -\sin \varphi_{1234} & 0 \\
\sin \varphi_{1234} & \cos \varphi_{1234} & 0 \\
0 & 0 & 1
\end{array}\right)
$$




$$
=\left(\begin{array}{ccc}
\cos \varphi_{1235} & -\sin \varphi_{1235} & 0 \\
\sin \varphi_{1235} & \cos \varphi_{1235} & 0 \\
0 & 0 & 1
\end{array}\right)\left(\begin{array}{ccc}
1 & 0 & 0 \\
0 & \cos \varphi_{2345} & \sin \varphi_{2345} \\
0 & -\sin \varphi_{2345} & \cos \varphi_{2345}
\end{array}\right)\left(\begin{array}{ccc}
\cos \varphi_{1245} & 0 & \sin \varphi_{1245} \\
0 & 1 & 0 \\
-\sin \varphi_{1245} & 0 & \cos \varphi_{1245}
\end{array}\right)
$$

where the cosines and sines are the following square roots of cross-ratios:

$$
\cos \varphi_{i j k l}=\sqrt{\frac{\zeta_{i l} \zeta_{j k}}{\zeta_{i k} \zeta_{j l}}}, \quad \sin \varphi_{i j k l}=\sqrt{\frac{\zeta_{i j} \zeta_{k l}}{\zeta_{i k} \zeta_{j l}}},
$$

and the exact sense of square roots may be, for instance, as follows: assume

$$
\zeta_{1}>\zeta_{2}>\zeta_{3}>\zeta_{4}>\zeta_{5}
$$

and take positive square roots. Computer algebra system Maxima ${ }^{1}$ was of great assistance in deriving and checking relation (10). Note again the nice parametrization: each of the matrices depends only on the $\zeta_{i}$ 's in the vertices of the corresponding tetrahedron. We emphasize this fact because this will be our goal in the more complicated situation of Section 5 , where our $\zeta_{i}$ 's will be $n \times n$ matrices.

A relation equivalent to (10) can be found in Kashaev's paper [5, Example 3].

\section{A construction of based $n$-dimensional spaces yielding pentagon relation}

Let now matrix entries in (4) - zeros, ones and asterisks - be themselves matrices of size $n \times n$. As long as we are not concerned about orthogonality, the construction of a pentagon relation goes practically the same way as in the beginning of Section 3 .

To be more exact, we now take for our space $V=12345$ the $3 n$-dimensional vector space of row vectors of length $5 n$ subject to $2 n$ linear dependencies. The row vectors will be written as

$$
\left(\begin{array}{lllll}
f_{1} & f_{2} & f_{3} & f_{4} & f_{5}
\end{array}\right),
$$

where each sans serif letter $\mathrm{f}_{i}$ denotes, in its turn, a row vector of $n$ components, and the linear dependencies are

$$
f_{1}+f_{2}+f_{3}+f_{4}+f_{5}=0, \quad f_{1} \zeta_{1}+f_{2} \zeta_{2}+f_{3} \zeta_{3}+f_{4} \zeta_{4}+f_{5} \zeta_{5}=0,
$$

where $\zeta_{i}$ are $n \times n$ matrices, with the condition that all their differences

$$
\zeta_{i j}=\zeta_{i}-\zeta_{j}
$$

are invertible.

We also introduce a notation for an $n \times 5 n$ matrix made of $n$ vectors of the form (11):

$$
\mathbf{f}=\left(\begin{array}{ccccc}
\mathrm{f}_{1}^{(1)} & \mathrm{f}_{2}^{(1)} & \mathrm{f}_{3}^{(1)} & \mathrm{f}_{4}^{(1)} & \mathrm{f}_{5}^{(1)} \\
\vdots & \vdots & \vdots & \vdots & \vdots \\
\mathrm{f}_{1}^{(n)} & \mathrm{f}_{2}^{(n)} & \mathrm{f}_{3}^{(n)} & \mathrm{f}_{4}^{(n)} & \mathrm{f}_{5}^{(n)}
\end{array}\right)=\left(\begin{array}{lllll}
f_{1} & f_{2} & f_{3} & f_{4} & f_{5}
\end{array}\right)
$$

where each $f_{i}=\left(\begin{array}{c}\mathrm{f}_{i}^{(1)} \\ \vdots \\ \mathrm{f}_{i}^{(n)}\end{array}\right)$ is, of course, an $n \times n$ matrix.

\footnotetext{
${ }^{1}$ Maxima, a computer algebra system, http://maxima. sourceforge.net.
} 
In analogy with Section 3, we introduce block matrices $\mathbf{f}_{i j k}$ with only $i$-th, $j$-th and $k$-th nonvanishing $n \times n$ matrix components. This means that, for instance,

$$
\mathbf{f}_{135}=\left(\begin{array}{lllll}
f_{1} & 0 & f_{1} \zeta_{51} \zeta_{35}^{-1} & 0 & f_{1} \zeta_{13} \zeta_{35}^{-1}
\end{array}\right) .
$$

We also assume the $n$ rows of such matrices to be linearly independent; they form then a basis in a corresponding space $i j k$, for instance, space 135 in the case of matrix (12). Then we can define $3 n \times 3 n$ matrices $P, Q, R, S$ and $T$ by the conditions (2) and (3), and these conditions again ensure the pentagon relation (5).

Let us write out explicit formulas for the matrix $P$ in the case where the bases in relevant spaces are chosen as

$$
\begin{array}{llll}
\mathbf{f}_{124}=\left(\begin{array}{lllll}
1 & \zeta_{14} \zeta_{42}^{-1} & 0 & \zeta_{12} \zeta_{24}^{-1}
\end{array}\right), & \mathbf{f}_{234}=\left(\begin{array}{lllll}
0 & 1 & \zeta_{24} \zeta_{43}^{-1} & \zeta_{23} \zeta_{34}^{-1}
\end{array}\right), \\
\mathbf{f}_{123}=\left(\begin{array}{llllll}
1 & \zeta_{13} \zeta_{32}^{-1} & \zeta_{12} \zeta_{23}^{-1} & 0
\end{array}\right), & \mathbf{f}_{134}=\left(\begin{array}{lllll}
1 & 0 & \zeta_{14} \zeta_{43}^{-1} & \zeta_{13} \zeta_{34}^{-1}
\end{array}\right)
\end{array}
$$

(compare this to formula (12) with $f_{1}$ set to identity matrix). Here we have left out the irrelevant (and zero) fifth matrix component of the vectors; recall also that, according to Remark 5, we identify matrices like $P$ with their direct sums with identity matrices. A direct calculation shows that the condition

$$
P\left(\begin{array}{l}
\mathbf{f}_{124} \\
\mathbf{f}_{234}
\end{array}\right)=\left(\begin{array}{l}
\mathbf{f}_{123} \\
\mathbf{f}_{134}
\end{array}\right)
$$

leads to the following explicit expression:

$$
P=\left(\begin{array}{cc}
1 & \zeta_{12} \zeta_{23}^{-1} \zeta_{34} \zeta_{42}^{-1} \\
1 & -\zeta_{14} \zeta_{42}^{-1}
\end{array}\right) .
$$

The problem of introducing a Euclidian metric making our operators $P, \ldots, T$ orthogonal is not so easy as in Section 3, and deserves a separate section.

\section{Making operators orthogonal}

We begin with analyzing how to introduce complex Euclidian metric in the space 1234 where operator $P$ acts nontrivially, aiming at making $P$ orthogonal; the same reasonings will apply to the other operators $Q, \ldots, T$, after obvious changes of indices. Space 1234 consists of $4 n$-row vectors

$$
\left(\begin{array}{llll}
f_{1} & f_{2} & f_{3} & f_{4}
\end{array}\right)
$$

with linear relations

$$
f_{1}+f_{2}+f_{3}+f_{4}=0 \quad \text { and } \quad f_{1} \zeta_{1}+f_{2} \zeta_{2}+f_{3} \zeta_{3}+f_{4} \zeta_{4}=0 .
$$

There are two conditions that can be considered separately, like we did with Conditions 2 and 3 in Section 3.

Condition 4. The decompositions

$$
1234=124 \oplus 234=123 \oplus 134
$$

must be orthogonal:

$$
124 \perp 234, \quad 123 \perp 134 .
$$


Condition 5. The bases within each of the spaces 124, 234, 123 and 134 must be orthonormal.

Condition 4 means that, for the matrices defined by formulas (13), the rows of matrix $\mathbf{f}_{124}$ must be orthogonal to the rows of matrix $\mathbf{f}_{234}$, and the rows of matrix $\mathbf{f}_{123}$ must be orthogonal to the rows of matrix $\mathbf{f}_{134}$.

We are searching for some complex Euclidian scalar product between vectors (15), (16), and with as good properties as possible. It turns out to be a good idea to take this scalar product in the following form:

$$
\left.\left(\begin{array}{llll}
\left(\mathrm{f}_{1}\right. & \mathrm{f}_{2} & \mathrm{f}_{3} & \mathrm{f}_{4}
\end{array}\right),\left(\begin{array}{llll}
\mathrm{g}_{1} & \mathrm{~g}_{2} & \mathrm{~g}_{3} & \mathrm{~g}_{4}
\end{array}\right)\right)=\mathrm{f}_{1} X \mathrm{~g}_{2}^{\mathrm{T}}+\mathrm{g}_{1} X \mathrm{f}_{2}^{\mathrm{T}}+\mathrm{f}_{3} Y \mathrm{~g}_{4}^{\mathrm{T}}+\mathrm{g}_{3} Y \mathrm{f}_{4}^{\mathrm{T}},
$$

where $X$ and $Y$ are $n \times n$ matrices to be found. The orthogonality conditions are re-written as follows:

$$
X+\zeta_{24} \zeta_{43}^{-1} Y \zeta_{24}^{-\mathrm{T}} \zeta_{12}^{\mathrm{T}}=0, \quad X \zeta_{32}^{-\mathrm{T}} \zeta_{13}^{\mathrm{T}}+\zeta_{12} \zeta_{23}^{-1} Y \zeta_{34}^{-\mathrm{T}} \zeta_{13}^{\mathrm{T}}=0
$$

where we use the notation $\zeta_{i j}^{-\mathrm{T}} \stackrel{\text { def }}{=}\left(\zeta_{i j}^{\mathrm{T}}\right)^{-1}$. Eliminating $Y$, we get the equation for $X$ :

$$
X=\zeta_{12} \zeta_{23}^{-1} \zeta_{43} \zeta_{24}^{-1} X \zeta_{12}^{-\mathrm{T}} \zeta_{24}^{\mathrm{T}} \zeta_{34}^{-\mathrm{T}} \zeta_{32}^{\mathrm{T}}
$$

Next good idea is to take all $\zeta_{i}$, and thus $\zeta_{i j}$, symmetric:

$$
\zeta_{i j}=\zeta_{i j}^{\mathrm{T}}
$$

Then equations (19) admit the following simple solution:

$$
X=\zeta_{12}, \quad Y=\zeta_{34} .
$$

Remark 8. Actually, equation (20), for symmetric $\zeta_{i}$ 's with invertible differences, can be solved completely, because it reduces to the form $X A B=B A X$. Our specific solution (21) seems, however, to be the best, because it also satisfies Condition 6 below.

Recall that the rows of the first matrix in (13) form a basis in the space 124, whose vectors we will write, for a moment, like

$$
\left(\begin{array}{lll}
f_{1} & f_{2} & f_{4}
\end{array}\right), \quad f_{2}=f_{1} \zeta_{14} \zeta_{42}^{-1}, \quad f_{4}=f_{1} \zeta_{12} \zeta_{24}^{-1} .
$$

Theorem 2. Scalar product (18) for vectors (22), given our solution (21), can be written in three equivalent forms:

$$
\begin{aligned}
& \left.\left(\begin{array}{lll}
\left(f_{1}\right. & f_{2} & f_{4}
\end{array}\right),\left(\begin{array}{lll}
g_{1} & g_{2} & g_{4}
\end{array}\right)\right) \\
& =\mathrm{f}_{1} \zeta_{12} \mathrm{~g}_{2}^{\mathrm{T}}+\mathrm{g}_{1} \zeta_{12} \mathrm{f}_{2}^{\mathrm{T}}=\mathrm{f}_{2} \zeta_{24} \mathrm{~g}_{4}^{\mathrm{T}}+\mathrm{g}_{2} \zeta_{24} \mathrm{f}_{4}^{\mathrm{T}}=\mathrm{f}_{4} \zeta_{41} \mathrm{~g}_{1}^{\mathrm{T}}+\mathrm{g}_{4} \zeta_{41} \mathrm{f}_{1}^{\mathrm{T}} .
\end{aligned}
$$

Proof. Direct calculation.

Remark 9. Formula (23) shows that the orientation of the triangle edges is relevant here: edges 12, 24 and 41, with this order of their vertices, are oriented as they must be as parts of the boundary of triangle 124, according to the classical algebraic-topological definition:

$$
\partial(i j k)=j k-i k+i j=i j+j k+k i .
$$

Theorem 3. There exists a unique complex Euclidian metric in the space 12345 satisfying the following conditions: 
(i) any two spaces corresponding to triangles ijk with disjoint interiors in Fig. 2 or 3 are mutually orthogonal,

(ii) the scalar product within a space $i j k$, where $i<j<k$, is given by any of the expressions in the second line of (23), with obvious changes $1 \mapsto i, 2 \mapsto j, 4 \mapsto k$.

Proof. First, we take one pentagon triangulation, for instance, the leftmost in Figs. 2 and 3, and define the scalar product within each of the three spaces corresponding to its triangles according to (ii), while setting - again by definition - these three spaces orthogonal to each other. Then, formulas (18), (21) and Theorem 2 (with proper changes of indices) show that the desired properties are preserved under any flip in Fig. 2 or 3.

In particular, we see that, in addition to Conditions 4 and 5 , the following condition holds.

Condition 6. The metric in a space $i j k$ depends only on $\zeta_{i}, \zeta_{j}$ and $\zeta_{k}$.

We now turn to Condition 5 and explain how to make the bases orthonormal within any space $i j k$, taking again 124 as example. We consider the Gramian - matrix of scalar products for the rows of the first matrix in (13). This Gramian is, according to (23),

$$
G_{124}=\left(\mathbf{f}_{124}, \mathbf{f}_{124}\right)=\zeta_{12} \zeta_{42}^{-1} \zeta_{14}+\zeta_{14} \zeta_{42}^{-1} \zeta_{12}=2 \zeta_{12} \zeta_{42}^{-1} \zeta_{14},
$$

here the last equality is an easy exercise.

Theorem 4. There exists an $n \times n$ matrix $c_{124}$ such that

$$
G_{124}=c_{124} c_{124}^{\mathrm{T}} \text {. }
$$

Proof. Gramian is, of course, symmetric: $G_{124}=G_{124}^{\mathrm{T}}$. So, there are many ways to find such matrix $c_{124}$ (and there are many such matrices for $n>1$ ). For instance, one can use the Takagi decomposition $[15,16]$ : for a complex symmetric $n \times n$ matrix $A$ there exists a unitary matrix $Q$ such that

$$
A=Q \Sigma Q^{\mathrm{T}}
$$

where $\Sigma=\operatorname{diag}\left(\sigma_{1}, \ldots, \sigma_{n}\right)$ with real nonnegative $\sigma_{i}$. Or, otherwise, the reader can show, as an exercise using the theory of equivalence and normal forms of matrices from textbook [3], that our nondegenerate symmetric matrix $G_{124}$ can even be represented as a squared, and also symmetric, matrix $c_{124}$.

Now the rows of matrix

$$
\mathbf{e}_{124}=c_{124}^{-1} \mathbf{f}_{124}
$$

give the desired orthonormal basis in 124 ; similarly in other spaces $i j k$. We get this way a pentagon relation for orthogonal matrices, which we formulate as the following theorem.

Theorem 5. Matrices $P^{\prime}, \ldots, T^{\prime}$ of the following transitions between bases:

$$
P^{\prime}\left(\begin{array}{c}
\mathbf{e}_{124} \\
\mathbf{e}_{234}
\end{array}\right)=\left(\begin{array}{l}
\mathbf{e}_{123} \\
\mathbf{e}_{134}
\end{array}\right), \quad \ldots, \quad T^{\prime}\left(\begin{array}{l}
\mathbf{e}_{125} \\
\mathbf{e}_{235}
\end{array}\right)=\left(\begin{array}{c}
\mathbf{e}_{123} \\
\mathbf{e}_{135}
\end{array}\right),
$$

and identified with larger matrices so that they acquire structure (4), are orthogonal and satisfy the pentagon relation $Q^{\prime} P^{\prime}=T^{\prime} S^{\prime} R^{\prime}$. Here bases $\mathbf{e}_{i j k}$ are defined according to formulas of type (26), and $c_{i j k}$ are any matrices satisfying conditions of type (25).

Explicit formulas for $c_{i j k}$ - and thus for $P^{\prime}, \ldots, T^{\prime}$ - are not so easy to obtain. Moreover, it may be more convenient, at least for an even $n$ and if we want to pass to Grassmann algebras in the style of Section 8 below, not to use orthonormal bases in spaces $i j k$, but bases consisting of isotropic vectors - vectors of length zero. This is what we are going to do, for $n=2$, in Section 6 . 


\section{Explicit formulas for $n=2$ and isotropic bases}

Let all $\zeta_{i}$ be symmetric $2 \times 2$ matrices, and consider again the basis $\mathbf{f}_{124}$ in the space 124 given by the first formula in (13) and the Gramian (24). For reasons that will be seen in Section 8, we want to construct a new basis

$$
\mathbf{g}_{124}=\left(\begin{array}{l}
\mathbf{g}_{124}^{(1)} \\
\mathbf{g}_{124}^{(2)}
\end{array}\right)=a_{124} \mathbf{f}_{124}
$$

such that

$$
\left(\mathbf{g}_{124}, \mathbf{g}_{124}\right)=\left(\begin{array}{ll}
0 & 1 \\
1 & 0
\end{array}\right)
$$

Condition (27) means, in particular, that both $\mathbf{g}_{124}^{(1)}$ and $\mathbf{g}_{124}^{(2)}$ have zero Euclidian lengths, i.e., are isotropic.

Denote the entries of $a_{124}$ and $G_{124}$ as

$$
a_{124}=\left(\begin{array}{cc}
x & y \\
x^{\prime} & y^{\prime}
\end{array}\right), \quad G_{124}=\left(\begin{array}{cc}
\alpha & \beta \\
\beta & \gamma
\end{array}\right),
$$

then (27) means the conditions

$$
\left(\begin{array}{ll}
x & y
\end{array}\right) G_{124}\left(\begin{array}{l}
x \\
y
\end{array}\right)=\left(\begin{array}{ll}
x^{\prime} & y^{\prime}
\end{array}\right) G_{124}\left(\begin{array}{l}
x^{\prime} \\
y^{\prime}
\end{array}\right)=0, \quad\left(\begin{array}{ll}
x & y
\end{array}\right) G_{124}\left(\begin{array}{l}
x^{\prime} \\
y^{\prime}
\end{array}\right)=1 .
$$

The solution to (28) is

$$
\left(\begin{array}{ll}
x & y
\end{array}\right)=c\left(\begin{array}{ll}
-\beta+\sqrt{\beta^{2}-\alpha \gamma} & \alpha
\end{array}\right), \quad\left(\begin{array}{ll}
x^{\prime} & y^{\prime}
\end{array}\right)=c^{\prime}\left(\begin{array}{ll}
-\beta-\sqrt{\beta^{2}-\alpha \gamma} & \alpha
\end{array}\right),
$$

with any $c$ and $c^{\prime}$ such that

$$
c c^{\prime}=\frac{1}{2 \alpha\left(\alpha \gamma-\beta^{2}\right)} .
$$

Next, we define, the same way, isotropic bases in all other spaces corresponding to our triangles $i j k$, and define matrices $\tilde{P}, \ldots, \tilde{T}$ by conditions

$$
\tilde{P}\left(\begin{array}{l}
\mathbf{g}_{124} \\
\mathbf{g}_{234}
\end{array}\right)=\left(\begin{array}{l}
\mathbf{g}_{123} \\
\mathbf{g}_{134}
\end{array}\right), \quad \ldots, \quad \tilde{T}\left(\begin{array}{l}
\mathbf{g}_{125} \\
\mathbf{g}_{235}
\end{array}\right)=\left(\begin{array}{l}
\mathbf{g}_{123} \\
\mathbf{g}_{135}
\end{array}\right) .
$$

Due to the orthogonality conditions of the form (17) and isotropy and normalization conditions of the form (27), the matrices $\tilde{P}, \ldots, \tilde{T}$ satisfy

$$
\tilde{P}^{\mathrm{T}} J \tilde{P}=\cdots=\tilde{T}^{\mathrm{T}} J \tilde{T}=J
$$

with

$$
J=\left(\begin{array}{llll}
0 & 1 & 0 & 0 \\
1 & 0 & 0 & 0 \\
0 & 0 & 0 & 1 \\
0 & 0 & 1 & 0
\end{array}\right) .
$$

After identifying these matrices with $6 \times 6$ matrices, so that they acquire the form (4), they satisfy the pentagon equation

$$
\tilde{Q} \tilde{P}=\tilde{T} \tilde{S} \tilde{R} .
$$


It remains to say that, for instance, $\tilde{P}$ is expressed the following way

$$
\tilde{P}=\left(\begin{array}{cc}
a_{123} & 0 \\
0 & a_{134}
\end{array}\right) P\left(\begin{array}{cc}
a_{124}^{-1} & 0 \\
0 & a_{234}^{-1}
\end{array}\right)
$$

in terms of $P$ given by formula (14).

Remark 10. The freedom in this construction is, first, in choosing the sign of square roots in (29) and, second, in choosing two values $c$ and $c^{\prime}$, obeying one condition (30), for every space $i j k$.

\section{Grassmann-Berezin calculus and pentagon relation in a Grassmann algebra}

A Grassmann algebra over the field $\mathbb{C}$ of complex numbers is an associative $\mathbb{C}$-algebra with unity, generators $x_{i}$ and relations

$$
x_{i} x_{j}=-x_{j} x_{i} .
$$

In particular, $x_{i}^{2}=0$, so an element of a Grassmann algebra is a polynomial of degree $\leq 1$ in each $x_{i}$. An even (odd) element in Grassmann algebra consists, by definition, only of monomials of even (resp. odd) total degrees.

The exponent is defined by the standard Taylor series. For example,

$$
\exp \left(x_{1} x_{2}+x_{3} x_{4}\right)=1+x_{1} x_{2}+x_{3} x_{4}+x_{1} x_{2} x_{3} x_{4} .
$$

For every Grassmann generator $x_{i}$, there are two derivations: left derivative $\frac{\partial}{\partial x_{i}}$ and right derivative $\frac{\overleftarrow{\partial}}{\partial x_{i}}$. These are $\mathbb{C}$-linear operations in Grassmann algebra defined as

$$
\frac{\partial}{\partial x_{i}}\left(x_{i} f\right)=f, \quad\left(f x_{i}\right) \frac{\overleftarrow{\partial}}{\partial x_{i}}=f
$$

where the element $f$ does not contain $x_{i}$; in this case also, of course,

$$
\frac{\partial}{\partial x_{i}} f=f \frac{\overleftarrow{\partial}}{\partial x_{i}}=0
$$

More generally, there are the following Leibniz rules: for an even or odd $f$,

$$
\frac{\partial}{\partial x_{i}}(f g)=\frac{\partial}{\partial x_{i}} f \cdot g \pm f \frac{\partial}{\partial x_{i}} g, \quad(g f) \frac{\overleftarrow{\partial}}{\partial x_{i}}=g \cdot f \frac{\overleftarrow{\partial}}{\partial x_{i}} \pm g \frac{\overleftarrow{\partial}}{\partial x_{i}} f
$$

namely, the plus is taken for an even $f$, and the minus for an odd $f$.

In some situations, the derivation in Grassmann algebra constitutes an analogue not to usual differentiation, but to integration. In such cases, it is called Berezin integral [1]; traditionally, Berezin integral is defined as the right derivative. In order to introduce the relevant notations, we also give its independent definition: it is a $\mathbb{C}$-linear operator in Grassmann algebra satisfying

$$
\int \mathrm{d} x_{i}=0, \quad \int x_{i} \mathrm{~d} x_{i}=1, \quad \int g h \mathrm{~d} x_{i}=g \int h \mathrm{~d} x_{i},
$$

if $g$ does not contain $x_{i}$; multiple integral is understood as iterated one, where the Fubini rule is applied according to the following model:

$$
\iint x y \mathrm{~d} y \mathrm{~d} x=\int x\left(\int y \mathrm{~d} y\right) \mathrm{d} x=1 .
$$


The Grassmann-algebraic pentagon relation we are dealing with in this paper looks as follows:

$$
\int \mathcal{W}_{1234} \mathcal{W}_{1345} \mathrm{~d} x_{134}=\text { const } \iiint \mathcal{W}_{1245} \mathcal{W}_{2345} \mathcal{W}_{1235} \mathrm{~d} x_{125} \mathrm{~d} x_{235} \mathrm{~d} x_{245}
$$

Here Grassmann variables $x_{i j k}$ (generators of the Grassmann algebra) are attached to all twofaces $i j k$ in Fig. 1; the Grassmann weight $\mathcal{W}_{i j k l}$ of a tetrahedron $i j k l$ depends on (i.e., contains) the variables on its faces, e.g., $\mathcal{W}_{1234}$ depends on $x_{124}, x_{234}, x_{123}$ and $x_{234}$. The integration in both sides of (33) goes over variables on inner faces (where the tetrahedra are glued together), while the result depends on the variables on boundary faces. Usually (compare, for instance, [8, formula (30)]), there appears also a numeric factor that cannot be included in a natural way in any $\mathcal{W}$; we denote it const in (33).

\section{Orthogonal operators from Grassmann-Gaussian exponents}

We denote Grassmann variables - generators of a Grassmann algebra - by letters $x$ and $y$ with subscripts. The operators of left multiplication by $x_{i}$ :

$$
f \mapsto x_{i} f
$$

and left differentiation w.r.t. $x_{i}$ :

$$
f \mapsto \frac{\partial}{\partial x_{i}} f
$$

satisfy fermionic anticommutation relations

$$
\left[x_{i}, x_{j}\right]_{+}=0, \quad\left[\frac{\partial}{\partial x_{i}}, \frac{\partial}{\partial x_{j}}\right]_{+}=0, \quad\left[x_{i}, \frac{\partial}{\partial x_{j}}\right]_{+}=\delta_{i}^{j},
$$

where the anticommutator of operators $A$ and $B$ is

$$
[A, B]_{+} \stackrel{\text { def }}{=} A B+B A .
$$

Operators (34) and (35) make thus a realization of fermionic creation-annihilation operators, and these operators generate a Clifford algebra, see [1, Chapter I].

The canonical transformations of the mentioned operators - linear transformations that preserve the anticommutation relations - are given by orthogonal linear operators [1, Chapter II]. Specifically, right now we are going to deal with algebras generated by two variables. For one algebra, called $\mathfrak{X}$, the generators are called $x_{1}$ and $x_{2}$, and the anticommutation relations for the abovementioned operators can be written in the following matrix form

$$
\left[\left(\begin{array}{c}
\frac{\partial}{\partial x_{1}} \\
x_{1} \\
\frac{\partial}{\partial x_{2}} \\
x_{2}
\end{array}\right),\left(\begin{array}{llll}
\frac{\partial}{\partial x_{1}} & x_{1} & \frac{\partial}{\partial x_{2}} & x_{2}
\end{array}\right)\right]_{+}=J
$$

where $J$ is our old matrix (31). The operators $\frac{\partial}{\partial x_{1}}, x_{1}, \frac{\partial}{\partial x_{2}}$ and $x_{2}$ are, of course, linearly independent, so a $4 \times 4$ matrix $M$ of an endomorphism of the subspace spanned by $\frac{\partial}{\partial x_{1}}, x_{1}, \frac{\partial}{\partial x_{2}}, x_{2}$, relative to this basis, preserves the anticommutation relations provided

$$
M^{\mathrm{T}} J M=J .
$$


We write such canonical transformation as

$$
\left(\begin{array}{c}
\frac{\partial}{\partial x_{1}} \\
x_{1} \\
\frac{\partial}{\partial x_{2}} \\
x_{2}
\end{array}\right) \mapsto M\left(\begin{array}{c}
\frac{\partial}{\partial x_{1}} \\
x_{1} \\
\frac{\partial}{\partial x_{2}} \\
x_{2}
\end{array}\right)
$$

One more algebra, called $\mathfrak{Y}$, is a copy of $\mathfrak{X}$, with generators called $y_{1}$ and $y_{2}$. Note that we introduce below $\mathbb{C}$-linear operators $\mathcal{A}, \mathcal{B}$ and $\mathcal{C}$ acting in the following spaces:

$$
\mathcal{A}: \mathfrak{X} \rightarrow \mathfrak{X}, \quad \mathcal{B}: \mathfrak{X} \rightarrow \mathfrak{Y}, \quad \mathcal{C}: \mathfrak{Y} \rightarrow \mathfrak{Y},
$$

while $A, B$ and $C$ will be simply $4 \times 4$ matrices.

We are now interested in canonical transformations obtained from Gaussian exponents. First, consider the operator $\mathcal{A}$ of multiplying by the following Gaussian exponent, where $a$ is a numeric coefficient:

$$
\mathcal{A}: \quad f \mapsto \exp \left(a x_{1} x_{2}\right) f
$$

As this operator obviously commutes with left multiplications by $x_{1}$ and $x_{2}$, while

$$
\frac{\partial}{\partial x_{1}}\left(\exp \left(a x_{1} x_{2}\right) f\right)=\exp \left(a x_{1} x_{2}\right)\left(\frac{\partial}{\partial x_{1}}+a x_{2}\right) f
$$

and

$$
\frac{\partial}{\partial x_{2}}\left(\exp \left(a x_{1} x_{2}\right) f\right)=\exp \left(a x_{1} x_{2}\right)\left(-a x_{1}+\frac{\partial}{\partial x_{2}}\right) f
$$

the following canonical transformation arises from pushing (34) and (35) from left to right through (36):

$$
\left(\begin{array}{c}
\frac{\partial}{\partial x_{1}} \\
x_{1} \\
\frac{\partial}{\partial x_{2}} \\
x_{2}
\end{array}\right) \mapsto \mathcal{A}^{-1}\left(\begin{array}{c}
\frac{\partial}{\partial x_{1}} \\
x_{1} \\
\frac{\partial}{\partial x_{2}} \\
x_{2}
\end{array}\right) \mathcal{A}=A\left(\begin{array}{c}
\frac{\partial}{\partial x_{1}} \\
x_{1} \\
\frac{\partial}{\partial x_{2}} \\
x_{2}
\end{array}\right), \quad \text { where } \quad A=\left(\begin{array}{cccc}
1 & 0 & 0 & a \\
0 & 1 & 0 & 0 \\
0 & -a & 1 & 0 \\
0 & 0 & 0 & 1
\end{array}\right) .
$$

Next, consider the operator $\mathcal{B}$ given by the following Berezin integral:

$$
\mathcal{B}: \quad f \mapsto \int \exp \left(b_{11} x_{1} y_{1}+b_{12} x_{1} y_{2}+b_{21} x_{2} y_{1}+b_{22} x_{2} y_{2}\right) f \mathrm{~d} x_{1} \mathrm{~d} x_{2} .
$$

Again, a simple calculation shows that pushing through (38) from left to right gives

$$
\left(\begin{array}{c}
\frac{\partial}{\partial y_{1}} \\
y_{1} \\
\frac{\partial}{\partial y_{2}} \\
y_{2}
\end{array}\right) \mapsto \mathcal{B}^{-1}\left(\begin{array}{c}
\frac{\partial}{\partial y_{1}} \\
y_{1} \\
\frac{\partial}{\partial y_{2}} \\
y_{2}
\end{array}\right) \mathcal{B}=B\left(\begin{array}{c}
\frac{\partial}{\partial x_{1}} \\
x_{1} \\
\frac{\partial}{\partial x_{2}} \\
x_{2}
\end{array}\right)
$$

where

$$
B=\frac{1}{\Delta}\left(\begin{array}{cccc}
0 & -b_{11} \Delta & 0 & -b_{21} \Delta \\
-b_{22} & 0 & b_{12} & 0 \\
0 & -b_{12} \Delta & 0 & -b_{22} \Delta \\
b_{21} & 0 & -b_{11} & 0
\end{array}\right), \quad \Delta=b_{11} b_{22}-b_{12} b_{21} .
$$


And our third operator $\mathcal{C}$ will be again like (36):

$$
\mathcal{C}: f \mapsto \exp \left(c y_{1} y_{2}\right) f .
$$

The analogue of (37) reads

$$
\left(\begin{array}{c}
\frac{\partial}{\partial y_{1}} \\
y_{1} \\
\frac{\partial}{\partial y_{2}} \\
y_{2}
\end{array}\right) \mapsto \mathcal{C}^{-1}\left(\begin{array}{c}
\frac{\partial}{\partial y_{1}} \\
y_{1} \\
\frac{\partial}{\partial y_{2}} \\
y_{2}
\end{array}\right) \mathcal{C}=C\left(\begin{array}{c}
\frac{\partial}{\partial y_{1}} \\
y_{1} \\
\frac{\partial}{\partial y_{2}} \\
y_{2}
\end{array}\right), \quad \text { where } \quad C=\left(\begin{array}{cccc}
1 & 0 & 0 & c \\
0 & 1 & 0 & 0 \\
0 & -c & 1 & 0 \\
0 & 0 & 0 & 1
\end{array}\right)
$$

The product $\mathcal{C B} \mathcal{A}$ of operators (40), (38) and (36) corresponds to the following Grassmann weight of tetrahedron 1234 :

$$
\mathcal{W}_{1234}=\exp \left(a x_{1} x_{2}+b_{11} x_{1} y_{1}+b_{12} x_{1} y_{2}+b_{21} x_{2} y_{1}+b_{22} x_{2} y_{2}+c y_{1} y_{2}\right),
$$

where we identify

$$
x_{1}=x_{124}, \quad x_{2}=x_{234}, \quad y_{1}=x_{123}, \quad y_{2}=x_{134},
$$

in the following sense:

$$
\mathcal{C B A}: \quad f \mapsto \int \mathcal{W}_{1234} f \mathrm{~d} x_{1} \mathrm{~d} x_{2}
$$

This operator makes, according to (37), (39) and (41), the following canonical transformation:

$$
\left(\begin{array}{c}
\frac{\partial}{\partial y_{1}} \\
y_{1} \\
\frac{\partial}{\partial y_{2}} \\
y_{2}
\end{array}\right) \mapsto(\mathcal{C B A})^{-1}\left(\begin{array}{c}
\frac{\partial}{\partial y_{1}} \\
y_{1} \\
\frac{\partial}{\partial y_{2}} \\
y_{2}
\end{array}\right) \mathcal{C B A}=C B A\left(\begin{array}{c}
\frac{\partial}{\partial x_{1}} \\
x_{1} \\
\frac{\partial}{\partial x_{2}} \\
x_{2}
\end{array}\right)
$$

We will need the explicit expression for $C B A$, which we also denote $W_{1234}$ :

$$
W_{1234}=C B A=\frac{1}{\Delta}\left(\begin{array}{cccc}
c b_{21} & b_{11}(a c-\Delta) & -c b_{11} & b_{21}(a c-\Delta) \\
-b_{22} & -a b_{12} & b_{12} & -a b_{22} \\
c b_{22} & b_{12}(a c-\Delta) & -c b_{12} & b_{22}(a c-\Delta) \\
b_{21} & a b_{11} & -b_{11} & a b_{21}
\end{array}\right) .
$$

Both sides of (33) can be regarded as kernels of integral operators acting on functions of three Grassmann variables. To be more exact, there are three "input" variables, corresponding to three triangles in the leftmost pentagon in Fig. 2 or 3, and three "output" variables, corresponding to the triangles in the rightmost pentagon in any of those figures. The mentioned integral operators are products of two or three operators of the form (43), respectively, for the l.h.s. or r.h.s. of (33), and each operator in these products corresponds to a tetrahedron.

Theorem 6. Let Gaussian exponents of the form (42) be taken for all Grassmann tetrahedron weights entering in (33) (of course, with its own parameters $a, \ldots, c$ for each tetrahedron). Then equality (33) holds, with a proper factor const, provided the five corresponding matrices (44) obey the pentagon relation in direct sum:

$$
W_{1345} W_{1234}=W_{1245} W_{2345} W_{1235} .
$$

Proof. It is known from general theory (see again [1]) that if a $\mathbb{C}$-linear operator $\mathcal{K}$ on Grassmann algebra corresponds to a (linear) canonical transformation $K$ in the manner like our $\mathcal{A}, \mathcal{B}$ and $\mathcal{C}$ corresponded to $A, B$ and $C$, then $\mathcal{K}$ is determined by $K$ up to a scalar factor. So, (33) follows if we take for $K$ first l.h.s., and then r.h.s. of (45). 


\section{A special case related to deformed exotic torsion}

In this section, we consider the case where all $\zeta_{i}$ 's have the form

$$
\zeta_{i}=\left(\begin{array}{cc}
\lambda_{i}+\mu_{i} & \mathrm{i} \mu_{i} \\
\mathrm{i} \mu_{i} & \lambda_{i}-\mu_{i}
\end{array}\right), \quad \mathrm{i}=\sqrt{-1} .
$$

Remark 11. All matrices of the form (46) commute. The usual Jordan form of matrix (46), if $\mu_{i} \neq 0$, is $\left(\begin{array}{cc}\lambda_{i} & 1 \\ 0 & \lambda_{i}\end{array}\right)$, see $[3]$.

Matrix $G_{124}$ has, according to (24), the same form (46), namely

$$
G_{124}=\left(\begin{array}{cc}
\lambda+\mu & \mathrm{i} \mu \\
\mathrm{i} \mu & \lambda-\mu
\end{array}\right), \quad \lambda=\frac{2 \lambda_{21} \lambda_{41}}{\lambda_{42}}, \quad \mu=\frac{2\left(\lambda_{41}^{2} \mu_{21}-\lambda_{21}^{2} \mu_{41}\right)}{\lambda_{42}^{2}}
$$

where we write, in the style of formula (8),

$$
\lambda_{i j}=\lambda_{i}-\lambda_{j}, \quad \mu_{i j}=\mu_{i}-\mu_{j} .
$$

Possible matrices $a_{124}$ are, according to Section 6, as follows:

$$
a_{124}=\frac{1}{2 \lambda} \operatorname{diag}\left(c_{124}, c_{124}^{-1}\right)\left(\begin{array}{cc}
2 & 2 \mathrm{i} \\
\lambda-\mu & -\mathrm{i}(\lambda+\mu)
\end{array}\right),
$$

where $c_{124}$ is an arbitrary nonzero constant.

Then we calculate the matrices $a_{234}, a_{123}$ and $a_{134}$ in the same way as we have done for $a_{124}$, only changing the indices as required, then the matrix $P$ according to (14), and finally $\tilde{P}$ according to (32). The result is

$$
\tilde{P}=\operatorname{diag}\left(c_{124}, c_{124}^{-1}, c_{234}, c_{234}^{-1}\right) \hat{P} \operatorname{diag}\left(c_{123}^{-1}, c_{123}, c_{134}^{-1}, c_{134}\right),
$$

where

$$
\hat{P}=\left(\begin{array}{cccc}
\frac{\lambda_{32} \lambda_{41}}{\lambda_{31} \lambda_{42}} & 0 & -\frac{\lambda_{32}}{\lambda_{31}} & 0 \\
-\frac{\lambda_{21} A}{\lambda_{31} \lambda_{32} \lambda_{42}^{2}} & 1 & -\frac{A}{\lambda_{31} \lambda_{42} \lambda_{43}} & -\frac{\lambda_{21} \lambda_{43}}{\lambda_{32} \lambda_{42}} \\
\frac{\lambda_{21} \lambda_{43}}{\lambda_{31} \lambda_{42}} & 0 & \frac{\lambda_{32}}{\lambda_{31}} & 0 \\
\frac{\lambda_{41} A}{\lambda_{31} \lambda_{42}^{2} \lambda_{43}} & 1 & -\frac{A}{\lambda_{31} \lambda_{42} \lambda_{43}} & \frac{\lambda_{41}}{\lambda_{42}}
\end{array}\right)
$$

and

$$
A=\left|\begin{array}{cccc}
1 & \lambda_{1} & \lambda_{1}^{2} & \mu_{1} \\
1 & \lambda_{2} & \lambda_{2}^{2} & \mu_{2} \\
1 & \lambda_{3} & \lambda_{3}^{2} & \mu_{3} \\
1 & \lambda_{4} & \lambda_{4}^{2} & \mu_{4}
\end{array}\right|
$$

(again, Maxima has helped much in these calculations).

The zeros in matrix (47), when compared with (44), show that $\zeta_{i}$ 's of the form (46) correspond to the case where

$$
a c-b_{11} b_{12}+b_{12} b_{21}=0 .
$$


This is exactly the case for the Grassmann tetrahedron weights already introduced in $[8,9]$. Recall that the starting point in these papers was Grassmann weights related to Reidemeister torsion of some exotic chain complexes, and then a deformation, like in [8, formula (23)], makes them

(up to a numeric factor) a Grassmann-Gaussian exponent for which the condition (48) can be easily checked. Actually, any Grassmann tetrahedron weight obeying (48) can be obtained from weights in [8,9] by a proper scaling $x_{i j k} \mapsto$ const $_{i j k} x_{i j k}$ of Grassmann generators. This shows that the present paper is about further nontrivial deformations of the exotic torsion weights.

\section{Acknowledgements}

The authors thank Rinat Kashaev for useful discussions, and the referees for careful reading of the first version of the paper and valuable comments.

\section{References}

[1] Berezin F.A., The method of second quantization, Pure and Applied Physics, Vol. 24, Academic Press, New York, 1966.

[2] Doliwa A., Sergeev S.M., The pentagon relation and incidence geometry, arXiv:1108.0944.

[3] Gantmacher F.R., The theory of matrices, Vol. II, Chelsea, New York, 1974.

[4] Kashaev R.M., On discrete three-dimensional equations associated with the local Yang-Baxter relation, Lett. Math. Phys. 38 (1996), 389-397, solv-int/9512005.

[5] Kashaev R.M., On matrix generalizations of the dilogarithm, Theoret. and Math. Phys. 118 (1999), 314-318.

[6] Kashaev R.M., Korepanov I.G., Sergeev S.M., The functional tetrahedron equation, Theoret. and Math. Phys. 117 (1998), 1402-1413, solv-int/9801015.

[7] Korepanov I.G., A dynamical system connected with inhomogeneous 6-vertex model, J. Math. Sci. 85 (1997), 1671-1683, hep-th/9402043.

[8] Korepanov I.G., Relations in Grassmann algebra corresponding to three- and four-dimensional Pachner moves, SIGMA 7 (2011), 117, 23 pages, arXiv:1105.0782.

[9] Korepanov I.G., Two deformations of a fermionic solution to pentagon equation, arXiv:1104.3487.

[10] Kuniba A., Sergeev S.M., Tetrahedron equation and quantum $R$ matrices for spin representations of $B_{n}^{(1)}$, $D_{n}^{(1)}$ and $D_{n+1}^{(2)}$, arXiv:1203.6436.

[11] Lickorish W.B.R., Simplicial moves on complexes and manifolds, in Proceedings of the Kirbyfest (Berkeley, CA, 1998), Geom. Topol. Monogr., Vol. 2, Geom. Topol. Publ., Coventry, 1999, 299-320, math.GT/9911256.

[12] Pachner U., P.L. homeomorphic manifolds are equivalent by elementary shellings, European J. Combin. 12 (1991), 129-145.

[13] Sergeev S.M., Quantization of three-wave equations, J. Phys. A: Math. Theor. 40 (2007), 12709-12724, nlin.SI/0702041.

[14] Sergeev S.M., Quantum curve in q-oscillator model, Int. J. Math. Math. Sci. 2006 (2006), Art. ID 92064, 31 pages, nlin.SI/0510048.

[15] Takagi T., On an algebraic problem related to an analytic theorem of Carathéodory and Fejér and on an allied theorem of Landau, Japan J. Math. 1 (1924), 83-93.

[16] Takagi T., Remarks on an algebraic problem, Japan J. Math. 2 (1925), 13-17. 